\title{
SÍNTESE E CARACTERIZAÇÃO DA PENEIRA MOLECULAR SBA-15: INFLUÊNCIA DA TEMPERATURA
}

\author{
G. M. de PAULA ${ }^{1}$, L. A. LIMA ${ }^{1}$ e M. G. F. RODRIGUES ${ }^{1}$ \\ ${ }^{1}$ Universidade Federal de Campina Grande, Unidade Acadêmica de Engenharia Química \\ E-mail para contato: gustafpaula@ hotmail.com
}

\begin{abstract}
RESUMO - Esse trabalho teve como objetivo sintetizar a peneira molecular SBA-15 sob diferentes temperaturas de síntese visando a obtenção de um material com melhores propriedades texturais. A peneira molecular foi sintetizada com tetraetil ortosilicato (TEOS) como fonte de sílica e o copolímero tribloco, poli-(oxido etileno)-poli-(oxido propileno)-poli-(oxido etileno) PEO-PPO-PEO como agente direcionador de estrutura, sob temperatura de $35^{\circ} \mathrm{C}$ ou $60{ }^{\circ} \mathrm{C}$ e agitação durante 24 horas. $\mathrm{O}$ material obtido, de $\mathrm{pH}$ próximo a 1 , foi submetido a tratamento hidrotérmico a $100^{\circ} \mathrm{C}$, em autoclave, durante 48 horas. O produto da síntese foi caracterizado por Difração de Raios-X (DRX) e Adsorção Física de $\mathrm{N}_{2}$ (BET). As análises de DRX mostraram que as sínteses foram obtidas com sucesso e a partir dos resultados do BET evidenciaram-se melhores propriedades texturais da síntese realizada a $35^{\circ} \mathrm{C}$.
\end{abstract}

\section{INTRODUÇÃO}

A peneira molecular SBA-15 é uma classe de silicato mesoporoso, caracterizada pelos poros tubulares uniformes e arranjo de canais ordenados hexagonalmente, estabilidade térmica mais elevada em comparação com M41S, elevado grau de ordenação estrutural, com área superficial elevada de $500-1000 \mathrm{~m}^{2} / \mathrm{g}$ (MEYNEN et al., 2009; ZHAO \& WAN, 1998).

A síntese da peneira molecular SBA-15 é, geralmente, realizada usando o tetraetilortosilicato (TEOS) como fonte de sílica e o copolímero tribloco, poli-(oxido etileno)-poli-(oxido propileno)-poli(oxido etileno) PEO-PPO-PEO como agente direcionador, dissolvidos em meio ácido sob agitação e aquecimento (ZHAO \& WAN, 1998). A síntese clássica da SBA-15 é realizada utilizando uma etapa de envelhecimento térmico seguida de um tratamento hidrotérmico.

Os parâmetros de síntese são extremamente importantes e podem ser divididos em dois grupos. Primeiro, fatores químicos que correspondem a reagentes, aditivos e solventes. Segundo, fatores físicos que correspondem a condições experimentais externas, tais como temperatura, agitação e duração das etapas. Dentre estes parâmetros, pode-se destacar a temperatura de síntese que desempenha um papel importante durante a formação da estrutura da peneira molecular SBA-15. As velocidades de hidrólise e condensação serão influenciadas como também o mecanismo e interação entre o surfactante (agente direcionador pluronic) e os precursores. 
Araújo (2013) observou que o tempo de síntese hidrotérmica em sistemas estáticos e dinâmicos influencia significativamente as propriedades texturais do material Al-SBA-15. Apesar de já haver estudos acerca desse assunto, uma melhor investigação sobre os melhores parâmetros de síntese da SBA-15 é extremamente importante para aperfeiçoar o processo de síntese da SBA-15. Dentro deste contexto, este trabalho tem como objetivo preparar peneira molecular SBA-15 variando a temperatura de síntese entre $35^{\circ} \mathrm{C}$ e $60{ }^{\circ} \mathrm{C}$ e avaliar a influência sobre as propriedades texturais, estruturais e morfológicas.

\section{MATERIAIS E MÉTODOS}

\subsection{Síntese da Peneira Molecular SBA-15}

A síntese da peneira molecular SBA-15 seguiu o procedimento baseado no procedimento descrito por ZHAO e WAN (1998), utilizando uma mistura reacional com composição molar: 1,0 Sílica: 0,017 P123: 5,7 HCl: $193 \mathrm{H}_{2} \mathrm{O}$. Inicialmente, dissolveu-se o surfactante Pluronic P123 (poli(óxido de etileno)-poli(óxido de propileno)- poli(óxido de etileno)) - Aldrich, em $\mathrm{HCl}$ concentrado, em seguida adicionou-se água, sob agitação e aquecimento a $35{ }^{\circ} \mathrm{C}$ ou $60{ }^{\circ} \mathrm{C}$, permanecendo por 4 horas para total dissolução do pluronic. Na sequência, o TEOS (tetraetil ortosilicato) foi adicionado, permanecendo sob agitação e aquecimento por 24 horas. Em seguida, o material foi levado à estufa para síntese hidrotérmica a $100^{\circ} \mathrm{C}$ durante 48 horas. Concluída esta etapa, o material obtido, com $\mathrm{pH}$ próximo a 1 , foi filtrado a vácuo e lavado com água destilada, sendo em seguida colocado para secar a $60^{\circ} \mathrm{C}$ por 24 horas. O material obtido foi calcinado, em mufla, com taxa de aquecimento de $10^{\circ} \mathrm{C} / \mathrm{min}$ por 7 horas, a uma temperatura de $550^{\circ} \mathrm{C}$. Os materiais sintetizados utilizando a mesma metodologia, apenas modificando a temperatura de síntese foram denominados: SBA-15(T35) e SBA-15(T60). A Figura 1 apresenta o diagrama da síntese da SBA-15.

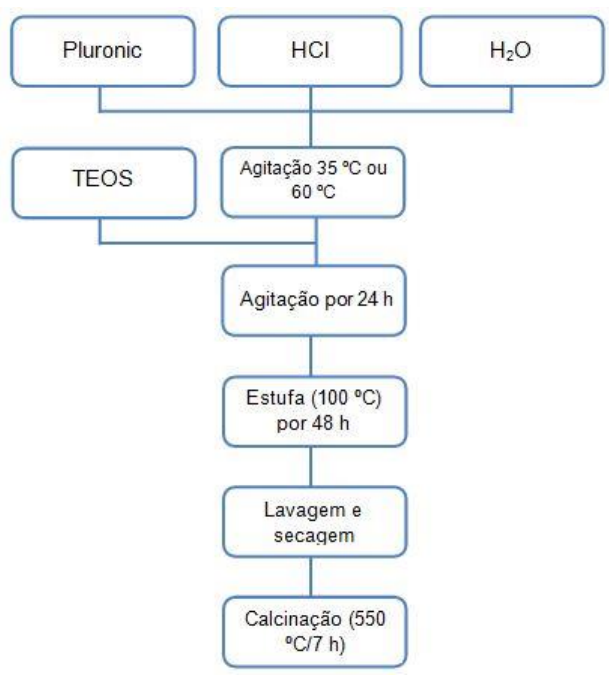

Figura 1 - Diagrama de síntese da peneira molecular SBA-15. 


\subsection{Caracterização}

Difração de Raios-X (DRX) - Foi utilizado o método do pó empregando-se um difratômetro Shimadzu XRD-6000 com radiação $\mathrm{CuK} \alpha$, tensão de $40 \mathrm{KV}$, corrente de $30 \mathrm{~mA}$, tamanho do passo de $0,0202 \theta$ e tempo por passo de $1,000 \mathrm{~s}$, com velocidade de varredura de $2^{\circ}(2 \theta) / \mathrm{min}$, com ângulo $2 \theta$ percorrido de 0,5 a $10^{\circ}$.

Adsorção Física de $\mathrm{N}_{2}$ (BET) - As características texturais das amostras analisadas foram investigadas mediante isotermas de adsorção/dessorção de $\mathrm{N}_{2}$ a $-196^{\circ} \mathrm{C}$, utilizando equipamento Micromeritics ASAP 2020. As isotermas de adsorção e dessorção de $\mathrm{N}_{2}$ foram obtidas na faixa de pressão relativa (P/Po) entre de 0,006 e 0,977.

Microscopia Eletronica de Varredura (MEV) - o aparelho utilizado foi um microscópio eletrônico de varredura da marca Philips, modelo XL30 EDAX. As amostras na forma de pó foram recobertas com uma fina camada de ouro por um metalizador e fixadas à porta amostras por uma fita adesiva de carbono.

\section{RESULTADOS E DISCUSSÃO}

Na Figura 1 estão apresentados os difratogramas das peneiras moleculares SBA-15 sintetizadas com temperaturas de síntese de $35^{\circ} \mathrm{C}$ e $60^{\circ} \mathrm{C}$.

a)

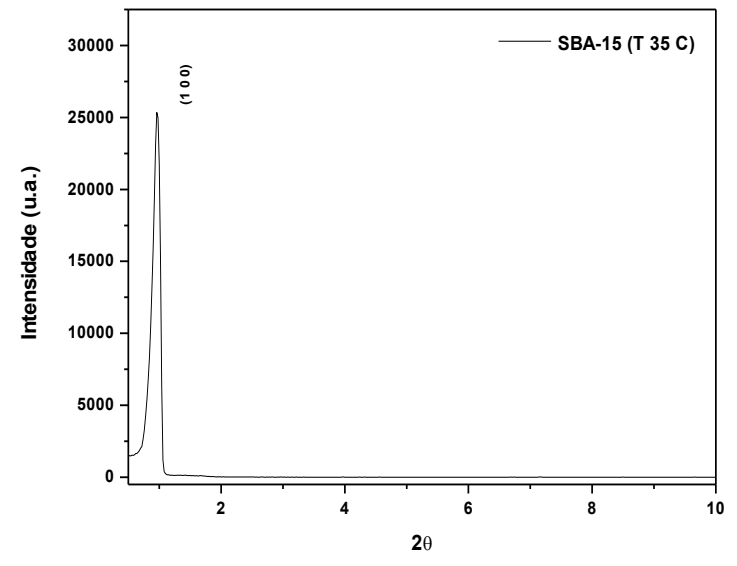

b)

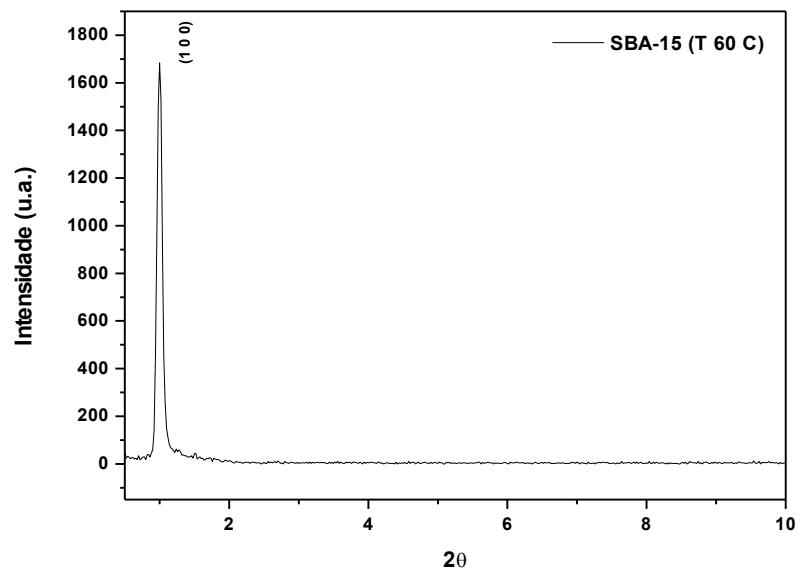

Figura 2 - Difratogramas das amostras: (a) SBA-15(T35); (b) SBA-15(T60).

A partir dos difratogramas apresentados na Figuras 1(a e b) verifica-se a presença do pico formado referente aos planos de Miller $\left(\begin{array}{lll}1 & 0 & 0\end{array}\right)$ característico da formação da estrutura mesoporosa. Os difratogramas apresentados também revelam que o aumento da temperatura de síntese de $35^{\circ} \mathrm{C}$ para $60^{\circ} \mathrm{C}$ produziu uma estrutura com menor grau de ordenação e cristalinidade, uma vez que a 
intensidade do pico característico da SBA-15 diminuiu de intensidade (RODRIGUES, 2011; LIMA et al., 2010; ZHAO \& WAN, 1998).

Na Figura 3 estão mostradas as imagens obtidas por microscopia eletrônica de varredura das peneiras moleculares SBA-15(T35) e SBA-15(T60).

a)

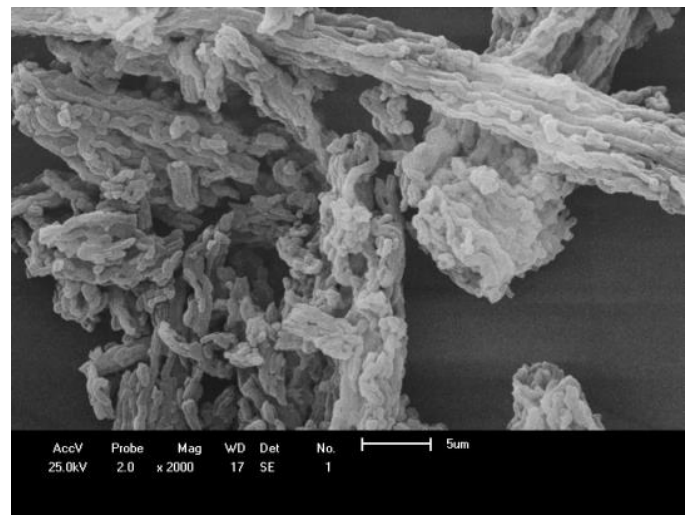

b)

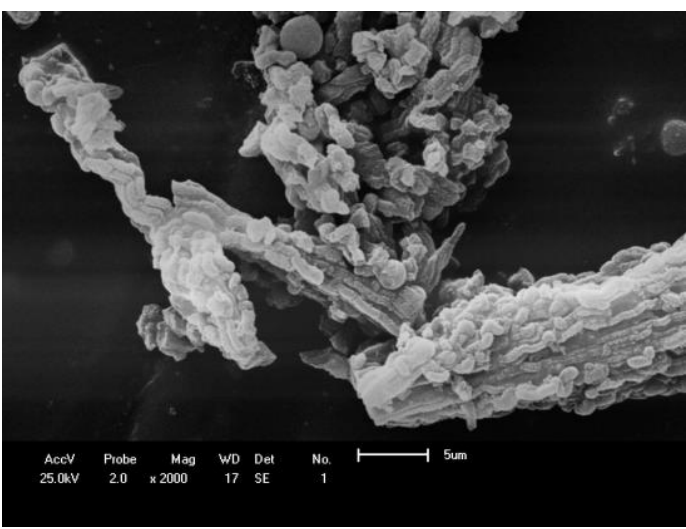

Figura 3 - Imagens das peneiras moleculares (a) SBA-15(T35) b) SBA-15(T60).

Observa-se que fibras de sílica com dimensões micrométricas são formadas a partir da adesão linear de nódulos de partículas sub-micrométricas. Em ambos os casos foram observadas fibras não uniformes, dando o aspecto de "colares de contas entrelaçados", estruturas similares às encontradas na literatura (KATIYAR et al., 2006; LIMA et al., 2010). Porém verifica-se uma diferença na morfologia da peneira molecular SBA-15(T35) e SBA-15(T60). A morfologia da peneira molecular SBA15(T35) parecer ser mais aglomerada que a morfologia da peneira molecular SBA-15(T60).

Na Figura 4 estão apresentadas as isotermas de adsorção-dessorção de $\mathrm{N}_{2}$ a $-196{ }^{\circ} \mathrm{C}$ referentes às peneiras moleculares SBA-15(T35) e SBA-15(T60).
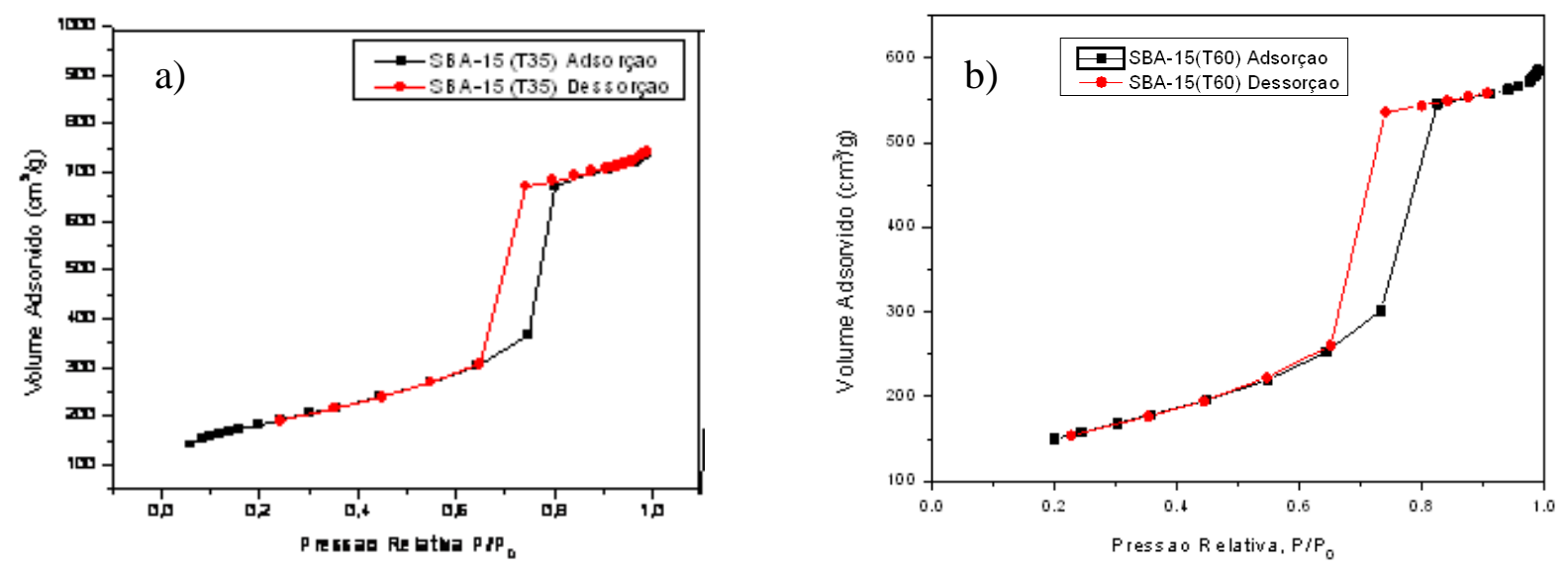

Figura 4 - Isotermas de adsorção-dessorção de $\mathrm{N}_{2}$ das peneiras moleculares a) SBA-15(T35); b) SBA-15(T60). 


\section{9 a 22 de outubro de 2014 \\ Florianópolis/SC}

As isotermas de adsorção/dessorção física de $\mathrm{N}_{2}$ a $-196^{\circ} \mathrm{C}$ apresenta perfil dentre os quatro encontrados em caracterização de catalisadores similar ao aspecto da isoterma do Tipo IV, relativa a materiais mesoporosos (SOAWANE e LUDOVICE, 2005; CHAREONPANICH et al., 2007). Três regiões podem ser distinguidas: a primeira, dada a baixas pressões relativas $\left(\mathrm{P} / \mathrm{P}_{0}<\right.$ 0,2 ) corresponde à adsorção de $\mathrm{N}_{2}$ na monocamada; a segunda inflexão dada entre $\mathrm{P} / \mathrm{P}_{0}=0,5-$ 0,8 ,ocorre a condensação capilar característico de materiais mesoporosos e a terceira $\left(\mathrm{P} / \mathrm{P}_{0}>0,9\right)$, pode ser atribuída a adsorção das multicamadas da superfície externa (SCHMIDT et al., 1995; SUVANTO e PAKKANEM, 2000).

A curva de dessorção apresenta "loop" de histerese do tipo $\mathrm{H}_{1}$ a pressões parciais $\left(\mathrm{P} / \mathrm{P}_{0}\right)$ entre $0,5-0,8$. Este comportamento corresponde a materiais porosos constituídos por cilindros abertos nas duas extremidades (SELVAM et al., 2001; KHODAKOV et al., 2005).

Na Tabela 1 estão apresentados os resultados das propriedades texturais das peneiras moleculares SBA-15(T35) e SBA-15(T60).

Tabela 1 - Propriedades texturais das amostras.

\begin{tabular}{|c|c|c|c|c|}
\hline Amostra & $\mathrm{S}_{\mathrm{BET}}\left(\mathrm{m}^{2} / \mathrm{g}\right)$ & $\mathrm{V}_{\mathrm{t}}\left(\mathrm{cm}^{3} / \mathrm{g}\right)$ & $\mathrm{dp}(\mathrm{nm})$ & $\mathrm{e}(\mathrm{nm})$ \\
\hline SBA-15 (T35) & 660 & 1,13 & 6,87 & 6,3 \\
\hline SBA-15 (T60) & 584 & 0,98 & 6,20 & 7,0 \\
\hline
\end{tabular}

Os valores encontrados para área superficial específica das peneiras moleculares: SBA-15(T35) é de $660 \mathrm{~m}^{2} / \mathrm{g}$ e SBA-15(T60) é de $584 \mathrm{~m}^{2} / \mathrm{g}$, e encontram-se dentro da faixa esperada conforme a literatura (MEYNEM et al., 2009). Realizando uma combinação das técnicas, difração de raios X e adsorção física de $\mathrm{N}_{2}$, foi possível calcular a espessura da parede como apresentado por Beck et al.,(1992). As peneiras moleculares: SBA-15(T35) e SBA-15(T60) apresentam valores de espessuras de paredes de $6,3 \mathrm{~nm}$ e $7,0 \mathrm{~nm}$. Esta espessura é responsável pela alta estabilidade hidrotérmica da SBA-15 comparada a outros materiais mesoporosos como MCM-41, MCM-48 e HMS (ZHAO et al., 1998).

Os resultados obtidos (Tabela1) para a peneira molecular SBA-15, para as duas temperaturas de síntese mostram que existem diferenças entre os valores de área superficial específica, volume de poros e diâmetro de poros. Os valores destes parâmetros encontrados para a peneira molecular SBA15(T35) são superiores aos valores encontrados para a peneira molecular SBA-15(T60). Porém o valor da espessura da parede da peneira molecular SBA-15(T35) é inferior ao valor da espessura da parede da peneira molecular SBA-15(T60). 


\section{CONCLUSÃO}

A influência da temperatura de síntese sobre as propriedades da peneira molecular SBA-15 foi investigada. É possível obter a peneira molecular nas duas temperaturas de síntese estudadas $\left(35^{\circ} \mathrm{C}\right.$ e $\left.60^{\circ} \mathrm{C}\right)$, porém a temperatura de síntese de $35^{\circ} \mathrm{C}$ conduziu a melhores resultados. Baseado nos resultados obtidos conclui-se que a temperatura de síntese afeta as propriedades da peneira molecular SBA-15, apresentando alteração nas propriedades texturais (área superficial específica, volume de poros e diâmetro de poros) e modificação na espessura da parede.

\section{AGRADECIMENTOS}

Os autores agradecem a ANP/PRH-25 pelas bolsas concedidas. Agradecem também ao apoio financeiro da PETROBRAS.

\section{REFERÊNCIAS}

ARAÚJO, N. F. Estudo das Variáveis Operacionais na Síntese da Peneira Molecular Mesoporosa Al-SBA-15, Seropédica, UFRRJ, 2013, Dissertação de Mestrado.

BECK, J. S., SOCHA, R. F., SHIHABI, D. S. \& VARTULI, J. C., U.S. Patent No 5,143,707, 1992.

DE WITTE, K.; BUSUIOC, A. M.; MEYNEN, V.; MERTENS, M.; BILBA, N.; VAN TENDELOO, G.; COOL, P.; VANSANT, E. F. Influence of the synthesis parameters of TiO2/SBA-15 materials on the adsorption and photodegradation of rhodamine-6G. Microporous and Mesoporous Materials, v. 110, p.100-110, 2008.

LIMA L. A. Desenvolvimento de catalisadores (Co/SBA-15) destinados a reação de FischerTropsch, Campina Grande, UFCG, 2009, Monografia.

LIMA, L. A.; RODRIGUES, J. J.; SILVA, V. J.; SOUSA, B. V.; RODRIGUES, M. G. F. Síntese, caracterização estrutural e morfológica da peneira molecular mesoporosa SBA-15, 56 Congresso Brasileiro de Cerâmicas, $1^{\circ}$ Congresso Latino-Americano de Cerâmicas, IX Brazilian Symposium on Glass and Related Materials, 2010

MEYNEN, V.; COLL, P.; VANSAT, E. F. Verified syntheses of mesoporous materials, Microporous and Mesoporous Materials. v.125, p.170-223, 2009.

RODRIGUES, J. J.; LIMA, L. A.; LIMA, W. S.; RODRIGUES, M. G. F.; FERNANDES, F. A. N. Fischer-Tropsch synthesis in slurry-phase reactors using Co/SBA-15 catalysts. Brazilian Journal of Petroleum and Gas, v. 5, p. 149-157, 2011. 
ZHAO, D.; WAN, Y. The Synthesis of Mesoporous Molecular Sieves. Studies in Surface and Catalysis, v. 268, cap.8, 1998.

SONWANE C.G.; LUDOVICE P.J. A note on micro- mesopores in the walls of SBA-15 and hysteresis of adsorption isotherms. Journal of Molecular Catalysis A: Chemical. v.238, p.135137, 2005.

CHAREONPANICH, M.; NANTA-NGERN, A.; LIMTRAKUL, J. Short-period synthesis of ordered mesoporous silica SBA-15 using ultrasonic technique. Journal of Materials Letters. v. 61, p. 5153, 2007.

SCHMIDT, R.; STOCKER, M.; HANSEN, E.; AKPORIAYE, D.; ELLESTAD, O. H. MCM-41: a model system for adsorption studies on mesoporous materials. Microporous and Mesoporous Materials. v. 3, p. 443-448, 1995.

SUVANTO, S.; PAKKANEN, T. A. Temperature programmed studies of Co on MCM-41 and $\mathrm{SiO}_{2 .}$. Journal of Molecular Catalysis A: Chemical. v. 164, p. 273 - 280, 2000.

SELVAM, P.; BHATIA S. K.; SONWANE C.; G. Recent advances in processing and characterization of periodic mesoporous MCM-41 silicate molecular sieves. Industrial \& Engineering Chemistry Research, v. 40, p. 3237 - 3261, 2001.

KHODAKOV, A. Y.; ZHOLOBENKO, V.L.; BECHARA, R.; DOMINIQUE, D. Impact of aqueous impregnation on the long-range ordering mesoporous structure of cobalt containing MCM-41 and SBA-15 materials. Microporous and Mesoporous Materials. v. 79, p. 29-39, 2005. 